\section{Soft X-ray Spectromicroscopy Development for Materials Science at the Advanced Light Source}

T. Warwick, H. Padmore, H. Ade, A.P. Hitchcock, E.G. Rightor, and B.P. Tonner Accelerator and Fusion Research Division

August 1996

Presented at the

First International Conference on Synchrotron Radiation in Materials Science, Chicago, IL, July 29-Aug. 2, 1996, and to be published in the Proceedings

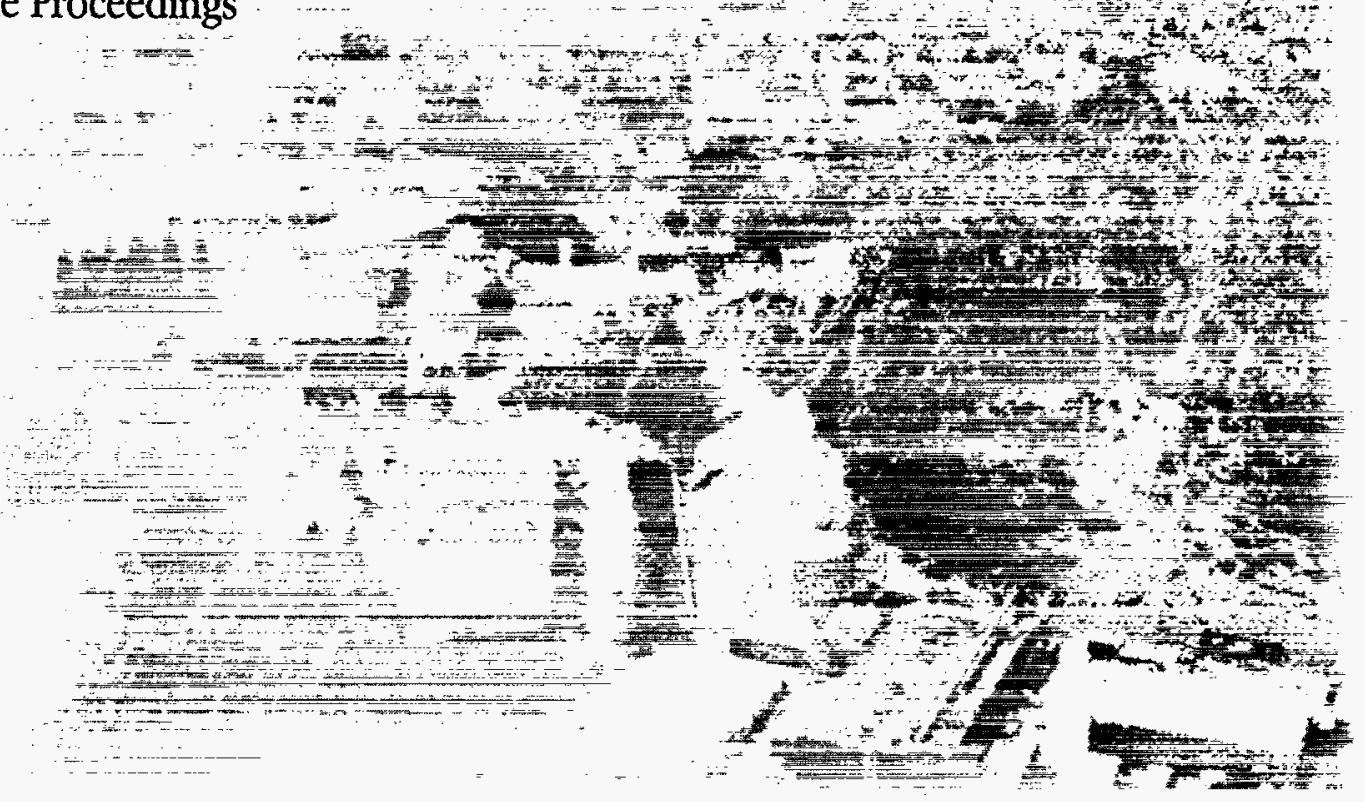

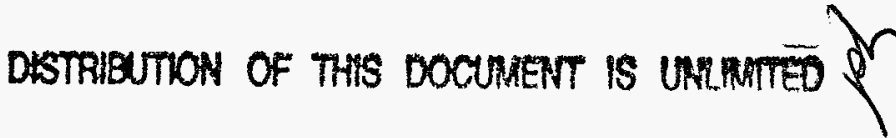

RECEIVED MOV 141996

OSTI 


\section{DISCLAIMER}

This document was prepared as an account of work sponsored by the United States Government. While this document is believed to contain correct information, neither the United States Government nor any agency thereof, nor The Regents of the University of California, nor any of their employees, makes any warranty, express or implied, or assumes any legal responsibility for the accuracy, completeness, or usefulness of any information, apparatus, product, or process disclosed, or represents that its use would not infringe privately owned rights. Reference herein to any specific commercial product, process, or service by its trade name, trademark, manufacturer, or otherwise, does not necessarily constitute or imply its endorsement, recommendation, or favoring by the United States Government or any agency thereof, or The Regents of the University of California. The views and opinions of authors expressed herein do not necessarily state or reflect those of the United States Government or any agency thereof, or The Regents of the University of California.

This report has been reproduced directly from the best available copy.

Ernest Orlando Lawrence Berkeley National Laboratory is an equal opportunity employer. 


\title{
SOFT X-RAY SPECTROMICROSCOPY DEVELOPMENT FOR MATERIALS SCIENCE AT THE ADVANCED LIGHT SOURCE*
}

\author{
Tony Warwick, Howard Padmore \\ Advanced Light Source, Lawrence Berkeley National Laboratory \\ University of California, Berkeley, California 94720 \\ Harald Ade \\ North Carolina State University \\ Raleigh, North Carolina 27695 \\ Adam P. Hitchcock \\ McMaster University \\ Hamilton, Ontario, L8S 4M1, Canada
}

Ed G. Rightor

Dow Texas Polymer Center

Freeport, TX 77541

Brian P. Tonner

University of Wisconsin

Milwaukee, WI 53211 
Recycled Paper 


\section{DISCLAIMER}

Portions of this document may be illegible in electronic image products. Images are produced from the best available original .document. 


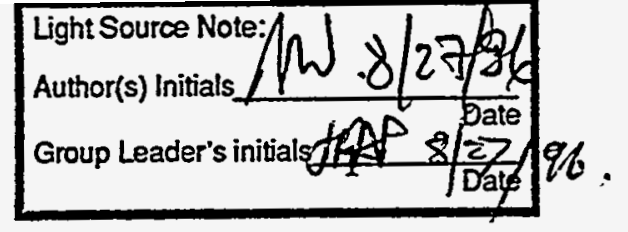

Soft X-Ray Spectromicroscopy Development for Materials Science at the Advanced Light Source.

Tony Warwick, Harald Ade*, Adam P. Hitchcock**, Howard Padmore, Ed. G. Rightor ${ }^{* * *}$ and Brian P. Tonner ${ }^{* * * *}$

Lawrence Berkeley National Laboratory, Berkeley, CA 94720, USA *North Carolina State University, Raleigh, NC 27695, USA

**McMaster University, Hamilton, Ontario, L8S 4M1, Canada ***Dow Texas Polymer Center, Freeport, TX 77541, USA ****University of Wisconsin, Milwaukee, WI 53211, USA

\section{Abstract}

Several third generation synchrotron radiation facilities are now operational and the high brightness of these photon sources offers new opportunities for $\mathrm{x}$-ray microscopy. Well developed synchrotron radiation spectroscopy techniques are being applied in new instruments capable of imaging the surface of a material with a spatial resolution smaller than one micron.

There are two aspects to this. One is to further the field of surface science by exploring the effects of spatial variations across a surface on a scale not previously accessible to $x$-ray measurements. The other is to open up new analytical techniques in materials science using $x$-rays, on a spatial scale comparable to that of the processes or devices to be studied.

The development of the spectromicroscopy program at the Advanced Light Source will employ a variety of instruments, some are already operational. Their development and use will be discussed, and recent results will be presented to illustrate their capabilities.

\section{Spectroscopy and microscopy techniques}

We consider spectroscopic measurements as 'spectromicroscopy' when the spatial features resolved are smaller than about 1 micron. There are two categories of microscope, and two spectroscopy techniques, which contribute to the spectromicroscopy program at the ALS. All of this work requires a synchrotron light source, and the advent of this and other third generation high brightness facilities is bringing these techniques into full practical use.

Scanning microscopes make a small spot of $x$-ray illumination on the sample surface. The size of the spot determines the spatial resolution which is usually at the diffraction limit of the x-ray optical system, which then has a correspondingly limited acceptance. A high brightness synchrotron source is required to fill the optics with sufficient intensity. With a stationary spot one can perform spectroscopy confined to the illuminated area. Images of the surface can be formed by scanning the spot (or the sample) over the area of interest and building up a picture by recording a signal, pixel by pixel. The signal will usually be derived from a 
specific spectral feature, e.g. the yield at a certain peak in the spectrum. Scanning to produce images is tedious, and it is difficult to study time dependent phenomena by this technique, but once the $x$-ray optics are implemented to produce a sub-micron spot the spatial resolution is guaranteed, regardless of the nature of the sample.

Photo-electron emission microscopes employ electron optics to form an image of the sample surface by collecting secondary electrons, or direct photo-electrons, through an electron optical column. Illumination requirements are less stringent thán with scanning microscopes; one needs only to put the photons on the sample surface over the field of view of the microscope. Electron optics can usually resolve better than $x$-ray optics, if the chromatic aberrations due to the spread of energy of the emitted electrons can be corrected. Generally the sample surface helps to form the focusing electric field so that it must be flat and conducting. The images can often be acquired at video rates so that time dependent phenomena are easy to watch.

$X$-ray Photoelectron Spectroscopy (XPS) is a well established discipline. Direct photo-electrons are measured for their yield, energy and spectral line shapes. The energy and lifetime of the core atomic excitation can be directly observed and its sensitivity to the structural and chemical environment of the emitting atom near the surface provides a lot of information. Chemical shifts of core level photo-emission spectra can be used to analyze the chemistry of surface structures. Angle resolved measurements show valence band dispersion and surface structure can be determined through photo-electron diffraction. Relative yield of photoelectrons from different species provide compositional information for materials science. The ALS spectromicroscopy program will employ this powerful technique on a sub-micron spatial scale.

$X$-ray Absorption Near-Edge Spectroscopy (XANES) is the second spectroscopic technique. Absorption spectra are often more difficult to interpret because the near edge structure depends on a valence state or molecular orbital into which the core electron is excited. Usually it is necessary to implement an auxiliary program of computation and measurement of known molecules and surfaces, to provide standard spectra against which those measured in a microscope can be compared. However, the results can be strikingly characteristic of the environment of the absorbing atom. In organic molecules, for example, the carbon atoms can be bound in many ways so that each molecular species has a carbon $\mathrm{K}$ edge absorption spectrum as its 'fingerprint' and the peaks in the absorption spectra can provide imaging contrast for spectromicroscopy. Ledge absorption spectra of magnetic materials provide a means of determining the magnetic moment contribution of an atomic species and a means to image the magnetization on a surface [1].

Figure 1. is an attempt to compare these spectromicroscopy techniques with some alternatives to synchrotron radiation. $X$-ray microscopes do not 
compete with electron microscopes for fine spatial resolution and some analytical techniques are available in an electron microscope. For example, electron energy loss spectroscopy (EELS) is analogous to XANES but with poorer spectral resolution and increased radiation damage to sensitive molecules. Since organics are an important category of samples, the issue of radiation damage and dose is crucial [2].

SEM/EDX is the identification of atomic species in an electron microscope image by fluorescence spectroscopic detection. This is a powerful imaging technique with the spatial resolution of an electron microscope, typicaly $10 \mathrm{~nm}$, but it does not give information on chemistry or structure.

Scanning Auger uses a focused electron beam and Auger electron detection, so that some chemical shifts can be measured, but the Auger signal to background ratio is small.

XPS in the lab can be improved in both spectral and spatial resolution by moving to a synchrotron source, where the high $x$-ray intensity allows narrow spectral selection for high energy resolution and the high brightness source leads naturally to small $x$-ray spots on the sample; spectromicroscopy follows.

Beamline development for spectromicroscopy at the ALS

Beamline 7.0.1 is an operational undulator beamline with high flux (typically $10^{12}$ photons/second) at high spectral resolution (typically 1 part in 8000 from $75 \mathrm{eV}$ to $1000 \mathrm{eV}$ ) [3]. It has been in use, principally for angle resolved photo-emission spectroscopy, for a year and a half, delivering photons into a spot on the sample 50 microns in diameter. It will also. serve zone plate scanning microscopes, which will demagnify the illumination to about 0.1 microns diameter for scanning spectromicroscopy. Figure 2. shows the beamline schematically. Two experiments can be illuminated interchangeably by means of a translating pair of mirrors. Two groups run their experiments together, trading shifts. Typically one group will be performing high intensity photo-emission studies, the other will be running samples in a microscope.

Beamline 7.3.1 is a bend magnet line under construction. Figure 3. shows the scheme. This line is designed to illuminate a sample surface for photo-electron emission microscopy, with operation from the carbon $\mathrm{K}$ edge to higher energies including the transition metal L edges around $700 \mathrm{eV}$. Carbon absorption spectroscopy and magnetic imaging using circular polarized light are the two key capabilities and the beamline provides spectral resolution of about $1 / 3000$ at the carbon edge to resolve XANES features and about $1 / 1000$ for the transition metals to measure spin orbit split $2 p$ line shapes. This line will also serve a large scale XPS instrument which is being developed in collaboration with the semiconductor industry. Structures fabricated on silicon wafers will be studied by XPS on a sub-micron spatial scale. 


\section{Scanning Transmission X-Ray Microscopy (STXM)}

A scanning transmission $X$-ray microscope has been operational for some time at beamline X1 at the NSLS [4]. The ALS spectromicroscopy program began with its own implementation of this technique. A Fresnel zone plate lens is employed to demagnify an illuminated pinhole into an $x$-ray spot at the diffraction limit of the optic. The sample is sectioned (typically $200 \mathrm{~nm}$ thick for organic samples to be studied at the carbon edge) and carried on a scanning $x / y$ stage which is encoded by capacitance micrometers and driven by piezo actuators in closed loop mode. This stage scans and the transmitted intensity is recorded to produce an absorption image. We have an intensity of about $10^{7}$ photons per second at the focus and we use a spectral bandwith of about $1 / 3000$. The zone plate is a diffractive lens with a first order diffraction efficiency of about $10 \%$. The zero order light is not focused and is blocked by an opaque central stop in the lens and by an aperture of the same size (the OSA), on the optical axis, close to the focus.

At the present time the zone plates in use at the ALS are 200 microns in diameter with an outer zone $80 \mathrm{~nm}$ wide, written in gold on a silicon nitride window $100 \mathrm{~nm}$ thick. This optic has a diffraction limited spot diameter of about $100 \mathrm{~nm}$ and the actual spot is measured at $150 \mathrm{~nm}$ FWHM. At this level the imaging capability matches the spatial resolution of the spectroscopic capability, which works in the following way. The microscope is focused by adjusting the longitudinal position of the zone plate until the image becomes sharp. This is done at a particular photon energy. The energy of the photons is then scanned through the absorption edge and the transmitted flux is recorded to obtain a XANES spectrum. As the photon energy is changed the zone plate lens must be moved longitudinally to maintain the focus, any accidental transverse motion of the lens during the scan causes motion of the spot on the sample and limits the size of features to be measured. If the spot walks off the feature during the spectral scan the measurement is useless. We have implemented a flexure mechanism to accomplish this focusing motion and a software algorithm is employed to remove the residual transverse errors by adjusting the transverse position of the sample during the spectral scan. This maintains the spot on target to the limit of the reproducibility of the mechanism, seen to be as good as $100 \mathrm{~nm}$. Control spectra must be measured with the sample removed to record the reprodicible wavelength dependent intensity changes in the beamline, which are due to geometry, carbon on mirrors and windows, and detector properties. Two detectors are installed. One is a silicon photo-diode with an oxide layer thinner than $8 \mathrm{~nm}$ for high efficiency. This detector is easy to use in current mode at full intensity. The second detector is a gas proportional counter with a thin $(100 \mathrm{~nm})$ silicon nitride window for efficient operation by photon counting at reduced intensity when sample damage by radiation is a concern. The gas in the counter is a mixture of $90 \%$ argon $10 \%$ carbon dioxide. The carbon dioxide quench gas provides a 
built in calibration feature for spectra at the carbon and oxygen edge. Pulse height discrimination helps to determine and reduce the contributions of higher order photons from the monochromator. The whole instrument is operated in a helium atmosphere to reduce absorption over the path from the beamline window to the detector, typically $6 \mathrm{~mm}$. Wet or dirty samples are acceptable. In principle, all edges above about $100 \mathrm{eV}$ are accessible, although nitrogen is difficult because of the nitride windows. Figure 4. shows the instrument.

Figure 5. shows some results of early measurements with this instrument. This is an extension of earlier work by one of us [5]. Fibers of kevlar are sectioned, imaged and measured for the absorption cross section at the carbon 1s, nitrogen $1 \mathrm{~s}$ and oxygen $1 \mathrm{~s}$ edges. Previous measurements were confined to the carbon edge. Images show preferential absorption above and below the horizontal plane through the center of the fiber, which is parallel to the plane of polarization of the $x$-rays, with an apparent cosine-squared dependence on the angle out-of-plane. The peak at $288.5 \mathrm{eV}$ is due to excitation of a 1s carbon electron into the $\pi^{*}$ orbital of the $\mathrm{C}=\mathrm{O}$ bond. The peak at $532 \mathrm{eV}$ is presumably due to excitation of a $1 \mathrm{~s}$ oxygen electron into the same $\pi^{*}$ orbital. In the kevlar molecule nitrogen is bound to carbon atoms from the carbonyl and from the aromatic group. The peaks at $403 \mathrm{eV}$ and $406 \mathrm{eV}$ are presumably due to excitation of a 1s nitrogen electron into the $\pi^{*}$ orbital of these bonds. In each case this $\pi^{*}$ absorption is enhanced when the electric vector of the photon is tangential to the circumference of the fiber, indicating that the bonds lie preferentially radially. The effect is so strong at the nitrogen edge that the two spectral peaks are not seen at locations in the fiber where the fiber radius vector is horizontal. At higher energies around $415 \mathrm{eV}$, above the nitrogen absorption edge, the polarization pattern is reversed, due to excitation into sigma orbitals. The ultimate goal of this study is to determine the degree of radial orientational order in various Kevlar fiber grades [6].

Other STXM measurements from the ALS are reported in reference [7].

\section{Demonstrating STXM analytical capabilities for polymer science}

Polyurethanes are considered a particularly versatile material because a small number of polymer components can be used to make a range of products with widely varying physical and chemical properties, the properties being controlled by the specific components as well as by the details of the polymerization processes. For this reason, methylenediphenyl-diisocyanate (MDI-) and toluene-diisocyanate (TDI-) based polyurethanes are produced industrially in large quantities. The overall composition of polyurethanes can vary widely, but generally involves polyether (derived from polyol precursor), polycarbamate $(\mathrm{R}-\mathrm{NH}(\mathrm{C}=\mathrm{O}) \mathrm{O}$ $\left.\mathrm{R}^{\prime}\right)$ and polyurea $\left(\mathrm{R}-\mathrm{NH}(\mathrm{C}=\mathrm{O}) \mathrm{NH}-\mathrm{R}^{\prime}\right)$ functionalities. The release of $\mathrm{CO} 2$ during the formation of the urea functionality is often exploited in the production of polyurethane foams as a blowing agent. The physical 
properties of certain slabstock polyurethane foams can be related to the microstructure, particularly the concentration, size distribution and composition of precipitates which segregate during foam formation. Spectromicroscopy techniques which can provide a quantitative chemical analysis of the matrix and precipitate composition, particularly the urea/carbamate ratio, are aiding product research. While IR or NMR spectroscopy can provide complementary information on the bulk composition of foams, these techniques do not have the spatial resolution to obtain chemical information on submicron precipitates. For this reason we are interested in exploring the capabilities of STXM based $x$-ray absorption spectromicroscopy for polyurethane microanalysis [2].

In STXM, the fully focused beam is sufficiently intense to cause chemical modifications to highly radiation sensitive polymers, even within the relatively small dose required for one spectral scan. The threshold for spectrally-detectable damage in the more sensitive (polyether-rich) polyurethanes occurs at an accumulated dose of approximately $5 \times 10^{6}$ photons. $\mu \mathrm{m}^{-2}$. Highly efficient use must be made of the allowable dose to accumulate a spectrum from a sub-micron region. Statistically, it should be possible to accomplish this with an efficient photon counting detector. The STXM technique is being developed towards this goal. Meanwhile, in order to obtain representative spectra of polyurethanes without detectable beam damage it was necessary to defocus the beam to a diameter of $>1 \mu \mathrm{m}$.

Figure 6. compares the $C 1$ S XAS spectra of polyurethane test samples (\#258, \#259 and \#260), acquired using the STXM spectro-microscopes at ALS and at NSLS (this program of research has made previos use of the original STXM at NSLS [4]). These samples were prepared by Werner Lidy (Dow Chemical) using procedures chosen to generate a well-known chemical composition (in particular a well-defined urea/carbamate ratio) in order to test the capability of X-ray absorption spectromicroscopy to carry out quantitative chemical analysis of urea/carbamate content. Electron microscopy shows that these samples are essentially homogeneous on spatial scales above $0.05 \mu \mathrm{m}$. In each case the spectra are the sum of several scans, each recorded with the photon beam defocused to a diameter of 10 $\mu \mathrm{m}$ in order to avoid radiation damage. Relative to the NSLS data the resolution of the ALS spectra appears to be slightly better, but the signal-tonoise ratio is somewhat worse. The match between the ALS and NSLS data is very good. The most significant differences in the spectral shape are the larger carbamate $\pi^{*} \mathrm{C}=\mathrm{O}$ signal at $290 \mathrm{eV}$ in the ALS spectrum of sample \#260 and the weaker $\pi^{*} \mathrm{C}=\mathrm{C}$ signal in sample \#258. These may partly be associated with the somewhat better energy resolution at the ALS, but could also reflect variability in the actual samples.

We have used the following procedure to derive quantitative compositional results for the urea/carbamate ratio in these samples. First, the spectral background is subtracted and all spectra are normalized, in the continuum above the absorption edge, to atomic oscillator strength 
intensities. Model spectra of the polyether, polyurea and polycarbamate components (recorded at the NSLS STXM) were also backgroundsubtracted and continuum-normalized. Each spectrum of the polyurethanes was then simulated as a sum of fractionally scaled model spectra, where the fractional scaling is equivalent to a mole fraction analysis. In order to generate the overall simulation, we first have isolated the aromatic component by assuming that the polyether component is that given by the stoichiometry predicted from the synthesis (see Table 1.). Figure 7. plots the results of isolating the aromatic component of the ALS spectra of the (\#258, \#259, \#260) samples, in comparison to the spectra of polyurea and polycarbamate models. After isolating the aromatic component it is clear that there are unique $\pi^{*} \mathrm{C}=\mathrm{O}$ features related to urea and carbamate functionality. In order to obtain internally consistent results, it was very. important to have accurate energy calibration (to within $0.05 \mathrm{eV}$ ) and careful normalization. Small adjustments were made to the energy scale linearity of the model spectra in order to obtain best match. The insert to Fig. 7. compares the isolated urea-carbamate $\pi^{*} \mathrm{C}=\mathrm{O}$ signal with curve fits used for the quantitative analysis. The peak heights for the urea and urethane signals from both ALS and NSLS are plotted against the composition predicted from the synthesis, in Fig. 8. Clearly the STXM X-ray absorption spectra can provide quantitative analysis with better than $10 \%$ compositional precision even in cases with 3 or more components. Better quality data will improve analytical precision. This can be readily obtained, even for highly radiation sensitive. samples, by averaging spectra acquired over morphologically similar micro structural regions. Ultimately it is expected that the analytical precision will be limited by systematic errors, such as the presence of unaccounted side products or additives, rather than by data quality.

Fig. 9. presents an image recorded at $285 \mathrm{eV}\left(\pi^{*} \mathrm{C}=\mathrm{C}\right)$ of a polyurethane foam, along with spectra recorded in the precipitate and in the adjacent matrix. Based on the continuum absorbance and the nearly featureless nature of images recorded at energies other than at the sharp spectral features, the section is uniform within $5 \%$ in both thickness and density. The images and spectra were recorded with the microscope fully focused, but with the photon intensity reduced about an order of magnitude from the maximum by de-tuning the undulator gap. After recording 5 or 6 spectra at slightly. different spots in the precipitate and matrix regions indicated in Fig. 9., images of this region were recorded at several photon energies to ensure that beam damage had not occurred. In this sample (\#250) there is a large difference in the matrix and precipitate spectra. Relative to the matrix, the precipitate spectrum has much stronger signals at the $\pi^{*} \mathrm{C}=\mathrm{C}(285.0 \mathrm{eV}) \pi^{*} \mathrm{C}=\mathrm{O}(289.0 \mathrm{eV})$, and at $286.3 \mathrm{eV}$. The signal at the latter energy is usually attributed to $C 1 s(C-R)$ to $\pi^{*} C=C$ transitions, although this feature is much more intense than counterpart features in model polymers or molecules of known structure [8]. While the full analysis of the precipitate requires further model compound studies, 
preliminary analyses based on sums of polyether, polyurea and polycarbamate spectra indicate the matrix is of the order of 50 to $60 \mathrm{~mol} \%$ polyether, with approximately equal contributions from both urea and carbamate as the aromatic functionality. In contrast, the precipitate is around $20 \mathrm{~mol} \%$ polyether, with the aromatic component being predominantly polyurea-like. The large signal at $286.3 \mathrm{eV}$ is as-yet unexplained.

Table 1.

Compositional Analysis of polyurethane test samples by $\mathrm{C}$ 1s spectromicroscopy.

\begin{tabular}{|c|c|c|c|c|}
\hline & \multicolumn{2}{|c|}{ FIT(a) } \\
\hline \multicolumn{2}{|c|}{ sample species } & prediction & NSLS & ALS \\
\hline 258 & $\begin{array}{l}\text { carbamate } \\
\text { urea } \\
\text { ether }\end{array}$ & $\begin{array}{l}.06 \\
.22 \\
.72\end{array}$ & $\begin{array}{l}.11 \\
.17 \\
(.72)\end{array}$ & $\begin{array}{l}.11 \\
.17 \\
(.72)\end{array}$ \\
\hline 259 & $\begin{array}{l}\text { carbamate } \\
\text { urea } \\
\text { ether }\end{array}$ & $\begin{array}{l}.11 \\
.11 \\
.78\end{array}$ & $\begin{array}{l}.18 \\
.10 \\
(.72)\end{array}$ & $\begin{array}{l}.16 \\
.12 \\
(.72)\end{array}$ \\
\hline 260 & $\begin{array}{l}\text { carbamate } \\
\text { urea } \\
\text { ether }\end{array}$ & $\begin{array}{l}.22 \\
.06 \\
.72\end{array}$ & $\begin{array}{l}.23 \\
.05 \\
(.72)\end{array}$ & $\begin{array}{l}.24 \\
.04 \\
(.72)\end{array}$ \\
\hline
\end{tabular}

(a) the relative peak heights of the urea and carbamate $\pi^{*} \mathrm{C}=\mathrm{O}$ signals derived from a curve analysis (see insert to Fig. 7.) were used to partition the 0.28 mole fraction of aromatic content, based on use of 0.72 as the mole fraction of the polyether component.

\section{Scanning Photo-Emission Microscopy (SPEM)}

Here we plan to implement XPS spectroscopy using a zone plate to create a small x-ray spot. This instrument is due to be operational in October 1996. The microscope functions in a way similar to STXM, there will be a capability for XANES using a similar mechanism, but implemented for ultra high vacuum. The sample will not be sectioned, rather the absorption will be measured by the total or partial yield of photo-electrons from the surface, detected as current-to-ground or by means of the hemispherical electron analyzer. However, the principle interest here is to collect photo-electron spectra from isolated sub-micron regions of the sample surface. For this mode of spectral acquisition there is no mechanical motion so that it should be easy to maintain the intrinsic spatial resolution. 
In order to include sample heating, cooling and orientation capabilities, we have decided to maintain the sample stationary in the SPEM design, once it is positioned for focus and for the region of interest. We will raster the zone plate lens in the illumination field for imaging. The zone plate (typically $200 \mathrm{~nm}$ diameter) is overfilled, the illumination is typically $1 \mathrm{~mm}$ FWHM, so that it will be possible to scan it $+/-50$ microns in $x$ and $y$ for small scale images. This is a new development and will bring complications, but the advantage of a large conventional sample manipulator are considered worth the trouble.

An additional concern is that the OSA must be carried on the scanning stage, which has limited weight capacity, so that the focal length must be built in. We plan to assemble zone plates together with their OSA into a pre-aligned unit, one zone plate/OSA combination with a fixed longitudinal separation will cover each absorption edge, through the XANES energy range and up to about $50 \mathrm{eV}$ above threshold for low kinetic energy photo-electron spectroscopy. For photo-electron spectroscopy with higher energy electrons the higher energy zone plate/OSA assemblies will be used to excite less tightly bound core levels. Figure 10. shows the interior layout of SPEM. This approach requires a plentiful supply of zone plates, and the ability to produce custom designs will be an advantage. At the present time there is no such plentiful supply. However there is a parallel project at LBNL to install an electron lithography facility capable of manufacturing the required zone plates. Production will commence late in 1996.

We expect to have $1 \times 10^{9}$ photons/second in the diffraction limited focal spot at $300 \mathrm{eV}$ (the spot can be as small as about $50 \mathrm{~nm}$ depending on the zone plate in use). At $700 \mathrm{eV}$ (for example) the flux is reduced, to about $8 \times 10^{7}$ photons/second, this is due both to the beamline design and to the fact that a diffraction limited system has a phase space acceptance inverseley proportional to the square of the photon energy, so that the undulator beam phase space increasingly exceeds the diffraction limited acceptance of the optical system as the photon energy is increased. More flux will be available by opening the pinhole and working with reduced spatial resolution.

The SPEM will use a large solid-angle, multi-channel photoemission spectrometer recently introduced by Physical Electronics Inc. (Phi Omega Analyzer). This electron spectrometer will collect sixteen energy channels simultaneously, from a solid-angle of up to 22 degrees half angle. Based on tests of this analyzer on the micro-focus stations of beamline 7 , we expect core-level photoemission count rates of $10^{4}$ with a bandpass of $0.5 \mathrm{eV}$. A total energy resolution (electron plus photon) of less than $0.08 \mathrm{eV}$ will be possible at reduced count rate. The reduction in count rate comes almost entirely from the decrease in analyzer pass energy, since the x-ray optics are designed to run at or near the maximum resolving power of the monochromator, when diffraction limited spot sizes are used. 
Photo-Electron Emission Microscopy (PEEM)

PEEM is a well developed technique [9]. Electron microscope technology can be applied to imaging with secondary electrons liberated by UV illumination to provide work function contrast images at the finest spatial resolution. When higher energy photons are used to excite the surface, XANES and XPS capabilities are added, but the larger spread of electron energies limits the resolution through chromatic aberration in the electron optics to a value of about $100 \mathrm{~nm}$. Further design effort is required to correct this problem and recover the resolution achievable with UV illumination. One approach is to make a narrow selection on the energy of the electrons used to form the image [10], this cuts down the microscope acceptance but introduces the capability to tune the selected electron energy to coincide with a photo-peak (at an intensity greatly reduced below that obtained using all the secondary electrons). Then the microscope can be used in XPS mode.

Otherwise the contrast mechanism is XANES. Electron mirror designs have been published [11] which will correct the chromatic aberrations while maintaining the high intensity imaging available when using the full spectrum of secondary electrons. Several groups around the world are implementing such schemes. High intensity images can be observed at video rates so that time dependent surface processes can be recorded with chemical state contrast. Alternatively, using circular polarized light (at beamline 7.3.1 for example) surface magnetization patterns can be observed.

Figure 11. shows an application of a PEEM at the ALS. Sub-micron particles are an unacceptable contaminant in semiconductor processing and as devices become smaller the tolerance of such particles becomes a major concern. XANES PEEM has been used here to image a test piece of silicon, which was deliberately contaminated with small particles ( 0.5 microns in this case). By illuminating the sample and scanning the photon energy through the aluminum $\mathrm{L}$ edge around $77 \mathrm{eV}$ the particles were made to emit electrons and the XANES spectra, measured from a small area in the image containing a particle, showed them to be alphaalumina. The requirement for a flat conducting sample is met here, and the insulating.particles are so small that their surface leakage currents prevent charging.

The program of PEEM measurements and instrument development will continue at beamline 7.3.1. Our present PEEM instrument will be employed, and new instrumentation will be developed. One particular application which comes naturally at beamline 7.3.1 is magnetic imaging and magnetic circular dichroism. The bend magnet radiation out of the orbit plane of the synchrotron is circularly polarized with opposite helicity from above and below the plane. By chopping the light with an aperture images and spectra of magnetized samples will be available which show the surface magnetization patterns and the atomic magnetic moments. 
Micro XPS for semiconductor structure analysis

Surface analysis for semiconductor processing will be an important application of soft $x$-ray spectromicroscopy. Any instrumentation to service this industry must include indexed wafer installation so that suspect features can be found for analysis. We are designing such a system to perform XPS analysis of silicon wafers with an $x$-ray spot 1 micron in diameter, at high intensity and moderate spectral resolution. This instrument will be installed at beamline 7.3.1, taking a small part of the available bend magnet angular distribution.

The $x$-ray spot is made by de-magnifying an illuminated pinhole, using grazing incidence mirrors. A pair of elliptical mirrors will be installed approximately $0.2 \mathrm{~m}$ upstream of the wafer, focusing horizontally and vertically. These mirrors form an image of an illuminated $15 \mu \mathrm{m}$ pinhole at a demagnification of 15 using an average deflection angle of 3.2 degrees. The mirrors must have an elliptical surface figure with slope deviations from the perfect shape by no more than $1 \mu$ rad r.m.s. along the illuminated length. A large indexed stage will translate the wafer into the spot so that a specific area can be analyzed. An optical alignment microscope is included to locate the features precisely. Figure 12. shows a plan view of this instrument, which is due to operate at the end of 1996.

\section{Summary}

Spectromicroscopy development at the ALS consists of the four projects outlined here. By early 1997 we expect to have each of these instruments running for surface science and materials analysis. The main application for STXM will be XANES spectromicroscopy of radiation sensitive organic molecules. SPEM will offer XPS spectromicroscopy in UHV for spatially resolved surface studies, e.g. spatially resolved chemisorption studies; it will also provide XPS analysis of submicron regions of structures fabricated off-line; such as adhesion layers in semiconductor structures, microcrystallites, composite structural materials. The microXPS facility will be devoted to analysis of features on silicon wafers. PEEM studies will emphasize magnetic imaging and magnetic circular dichroism.

\section{Acknowledgement}

This status report represents the continuing hard work of many people, within the Experimental Systems Group at the Advanced Light Source. The NSLS STXM has been build and maintained by the Stony Brook $x$-ray microscopy groups of J. Kirz and C. Jacobsen. HA acknowledges support through a NSF Young Investigator Award (DMR-9458060) and a grant from Dow Chemical Corporation. The Kevlar samples have been provided by B. Hsiao and S. Subramoney from DuPont.

This work was supported by the Director, Office of Energy Research, Office of Basic Energy Sciences, Materials Sciences Division of the U.S. Department of Energy, under Contract No. DE-AC03-76SF00098 


\section{References}

[1] J.Stohr, M.Samant and Y.Wu, Science 259(1993)658

[2] E.G.Rightor, A.P.Hitchcock, H.Ade, R.D.Leapman, S.G.Urquhart, A.P.Smith, G.Mitchell, D.Fischer, H.J.Shin and T.Warwick, J. Phys. Chem., to be published.

[3] T.Warwick, P.Heiman, D.Mossessian,W.McKinney and H.Padmore, Rev. Sci. Instr. 66(1995)2037

[4] S.Williams, C.Jacobsen, J.Kirz, S.Wirick, X.Zhang, H.Ade and M.Rivers, Rev. Sci. Instr. 66(1995)1271

[5] H.Ade and B.Hsiao, Science 262(1993)1427

[6] A.P.Smith and H. Ade, Appl. Phys. Lett., to be published.

[7] B.P.Tonner, D.Dunham, T.Droubay, J.Kikuma, J.Denlinger, E.Rotenberg and A.Warwick, J. Elec. Spectrosc. Rel. Phenom. 75(1995)309

[8] S.G.Urquhart, A.P.Hitchcock, E.G.Rightor, R.D.Priester and R.D.Leapman, J. Polymer Science B33 (1995) 1593, 1603.

[9] W.Telieps and E.Bauer, Ultramicroscopy 17(1985) 57

[10] B.P.Tonner, D.Dunham, T.Droubay and M.Pauli, J. Elec. Spectrosc. Rel. Phenom. (this issue)

[11] G.F.Rempfer, J. Appl. Phys. 67(1990)6027

\section{Figure 1.}

The regime of spatial and spectral resolution for soft $x$-ray spectromicroscopy at a synchrotron radiation source, and some related techniques.

\section{Figure 2.}

Beamline 7.0.1 is one of the first undulator lines to become operational at the ALS. It employs a water cooled spherical grating monochromator to deliver the full intensity photon beam at high resolution (1/8000). Small spot XPS measurements and spectromicroscopy experiments are performed interchangeably on two end station branches. 


\section{Figure 3.}

Beamline 7.3.1 provides illumination for photo-electron emission microscopy using linear or circular polarized light, it is also the source for an XPS station in which spectromicroscopy experiments are performed on silicon structures from the semiconductor manufacturing industry.

\section{Figure 4.}

A Fresnel zone plate is used to form the $\mathrm{x}$-ray spot for STXM. The first order light focuses on the sample, unfocused zero order radiation must be blocked by the zone plate central stop and by an order sorting aperture close to the focus. The focal length is proportional to the photon energy. All motions are motorized so that the microscope is fully operational in its helium enclosure.

\section{Figure 5.}

Images and spectra of sectioned kevlar fibers measured at the three important absorption edges. Polarization dependent absorption is seen as a cosine-squared dependence on the angle out of the horizontal plane of polarization of the photons (see text).

\section{Figure 6.}

XAS spectra measured for three polyurethane test samples from Dow Chemical in STXM at NSLS and at ALS. The two instruments are seen to give the same result (see text).

Figure 7.

Comparison of the aromatic component of the measured absorption spectra of the three test samples, for extraction of the carbamate/urea ratio (see text).

Figure 8.

Comparison of the quantitative measurement of the carbamate/urea ratio from STXM peak height analysis with that known from synthesis, for the three polyurethane test samples.

\section{Figure 9.}

An image of a region of polyurethane foam (\#250) containing precipitate particles. Spectra are shown for matrix and precipitates, their differences yield quantitative analyses of the polymer composition (see text).

\section{Figure 10.}

Side view of the SPEM interior showing the zone plate/OSA array on the scanning stage, the large conventional sample manipulator, and the lens of the analyzer in the background. 
Figure 11.

XANES spectromicroscopy by photo-electron emission microscopy (PEEM) of 0.5 micron particles on a silicon wafer. By scanning the photon energy and recording the brightness of small areas of the image a XANES

spectrum shows the particles to be made of alpha-alumina. This study was perfomed in collaboration with C.R. Brundle with samples provided by SEMATECH.

\section{Figure 12.}

Plan view of the micro XPS instrument, showing the elliptical mirrors, the indexed wafer stage, sample loading hardware and the hemispherical electron analyzer. 


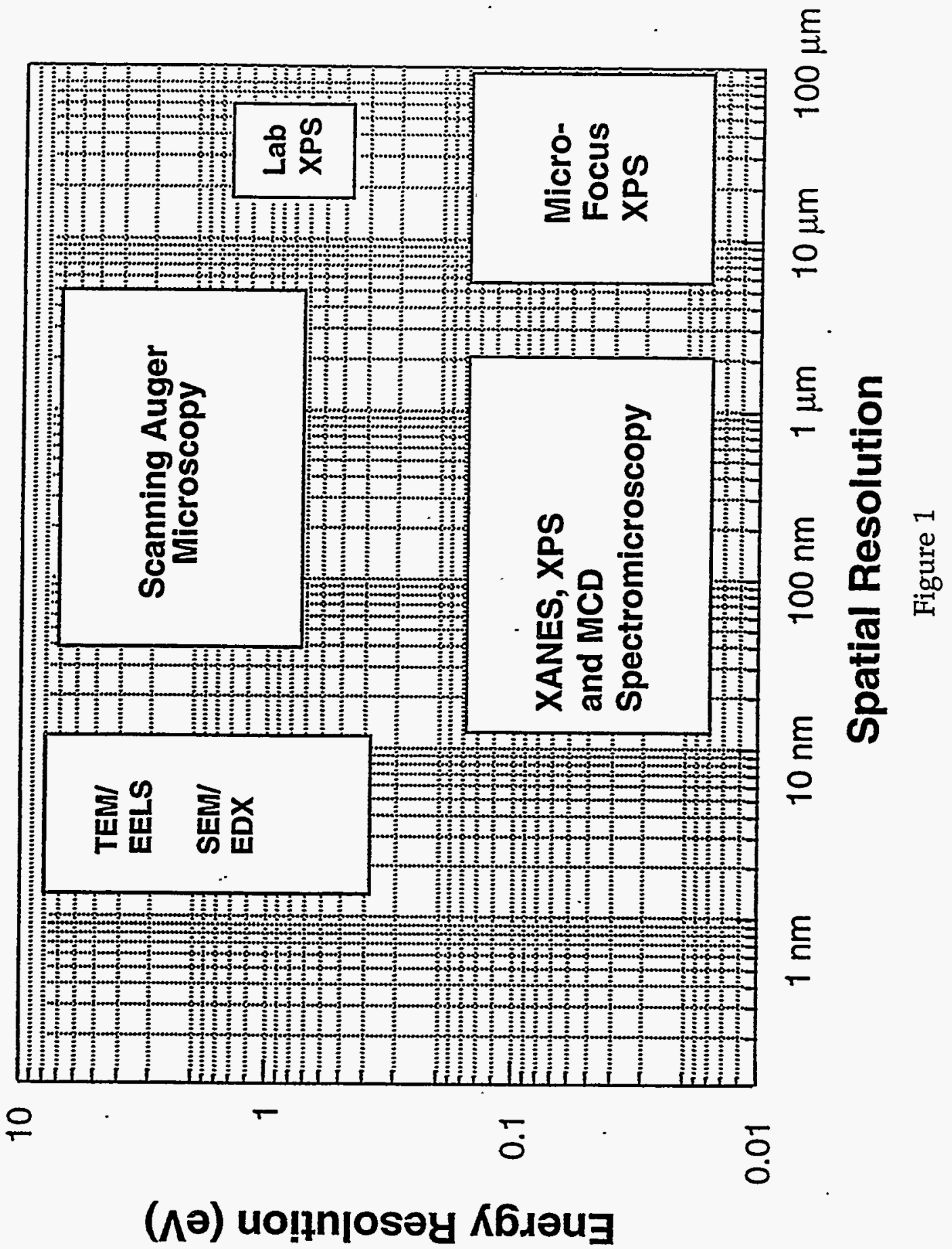



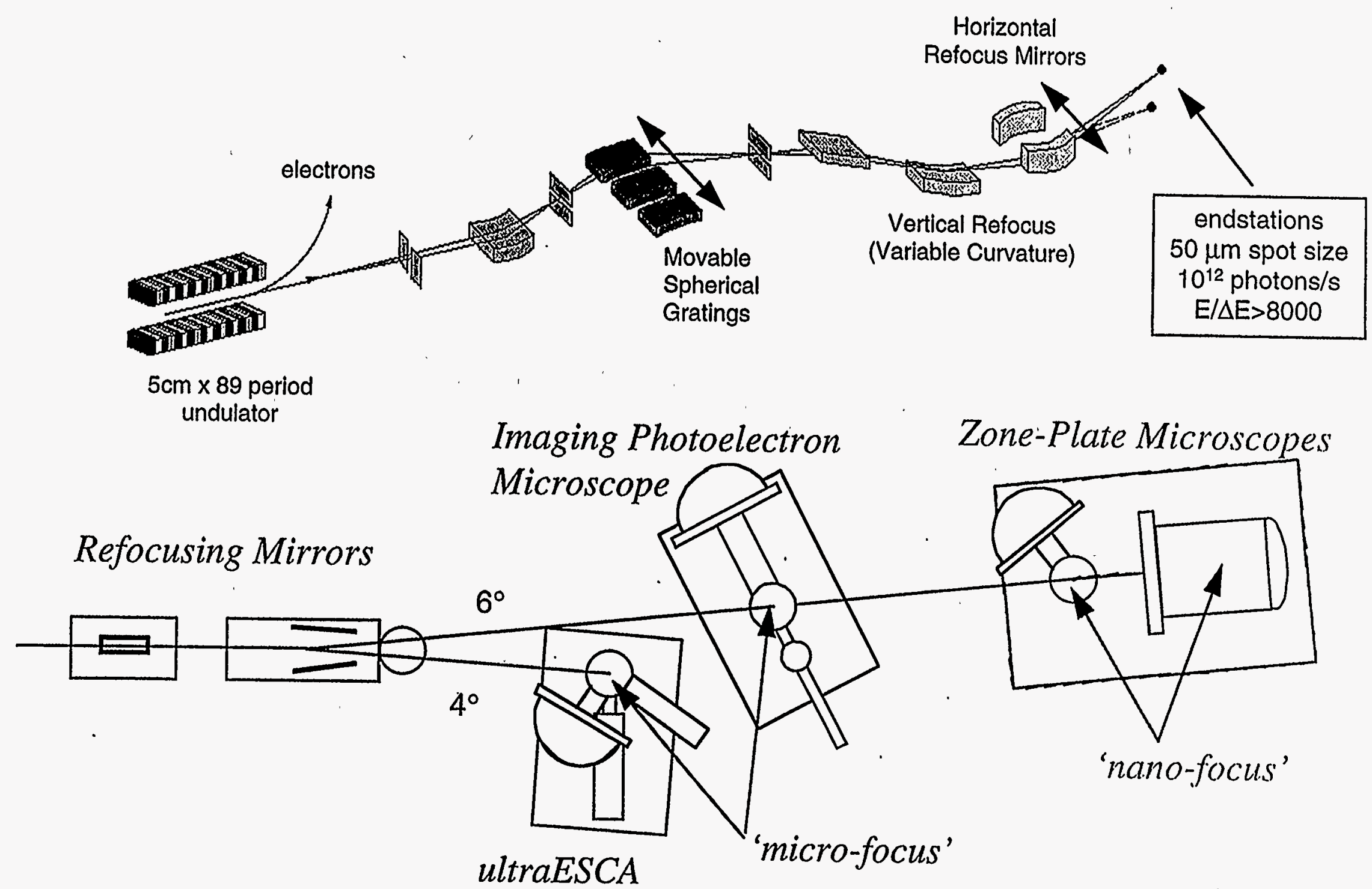

Figure 2 


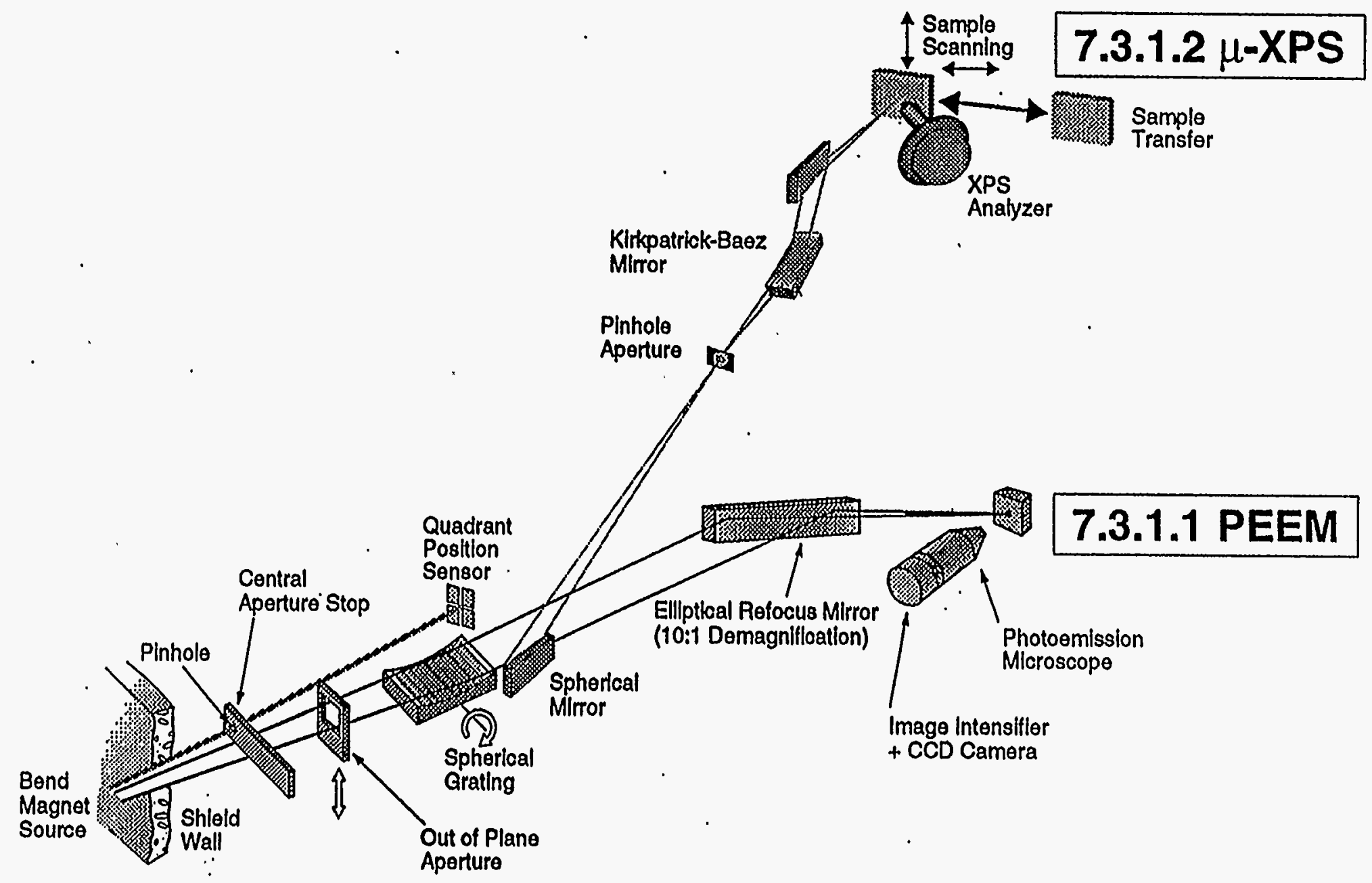

Figure 3 

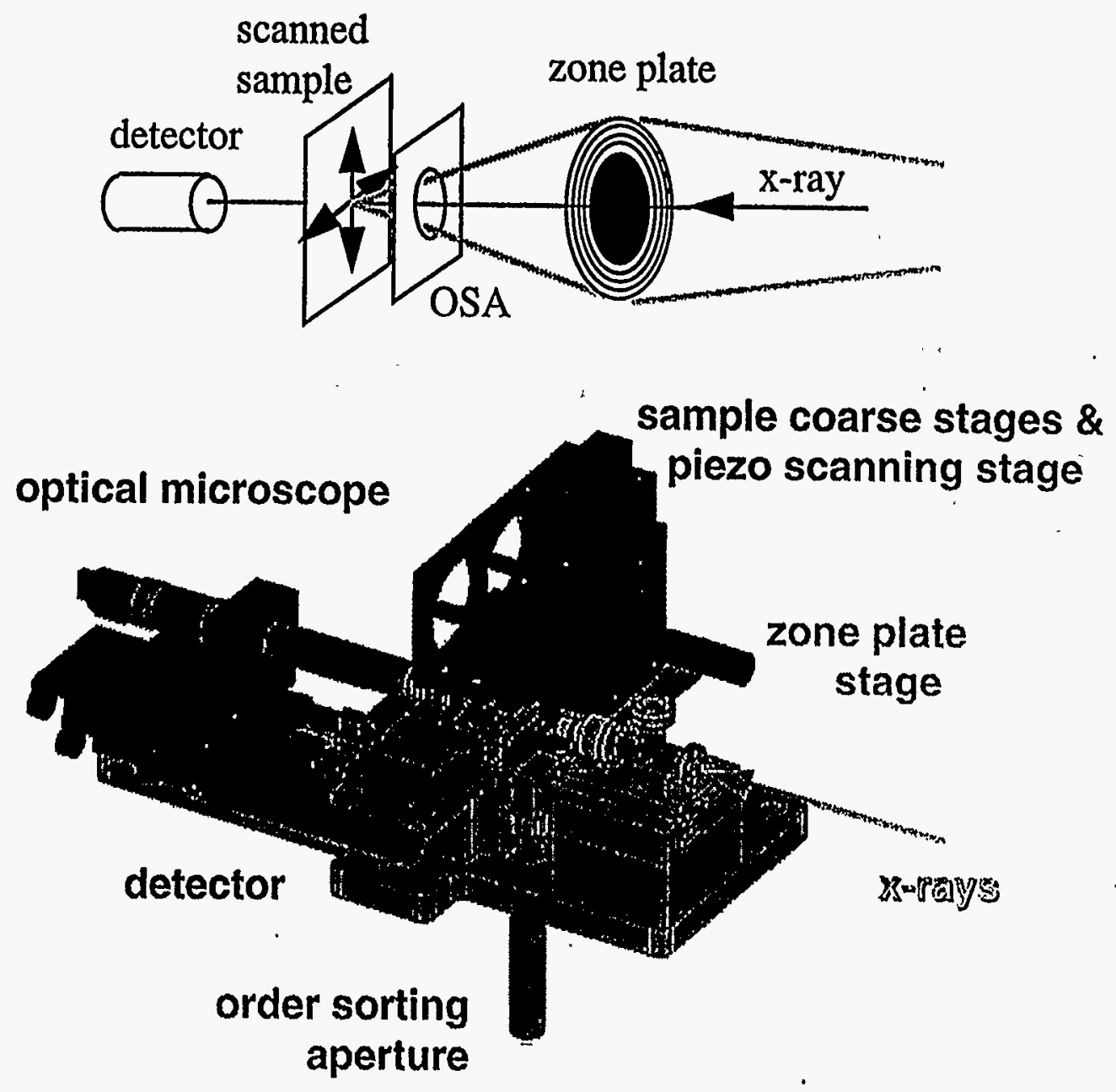

Figure 4 

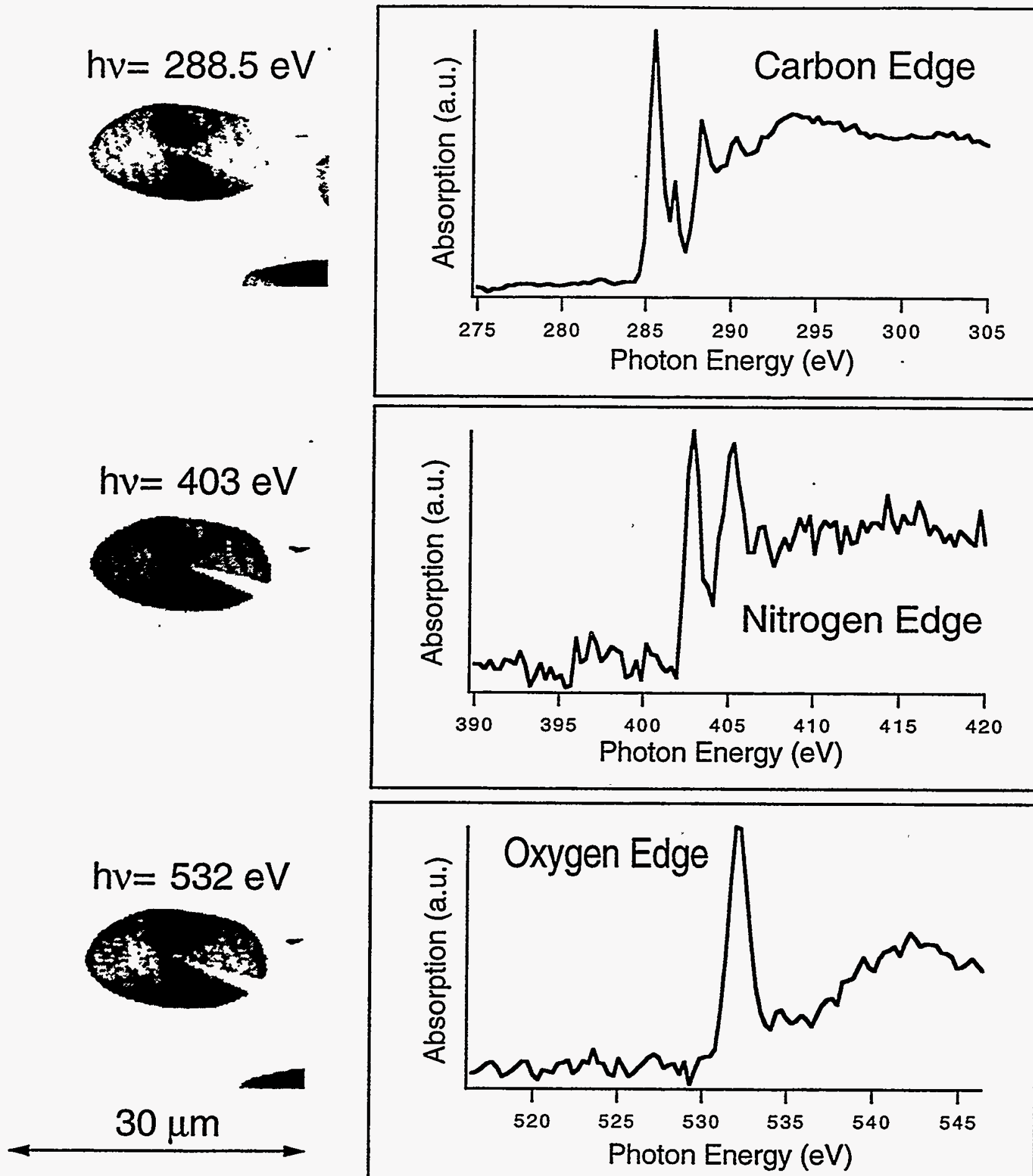

Figure 5 


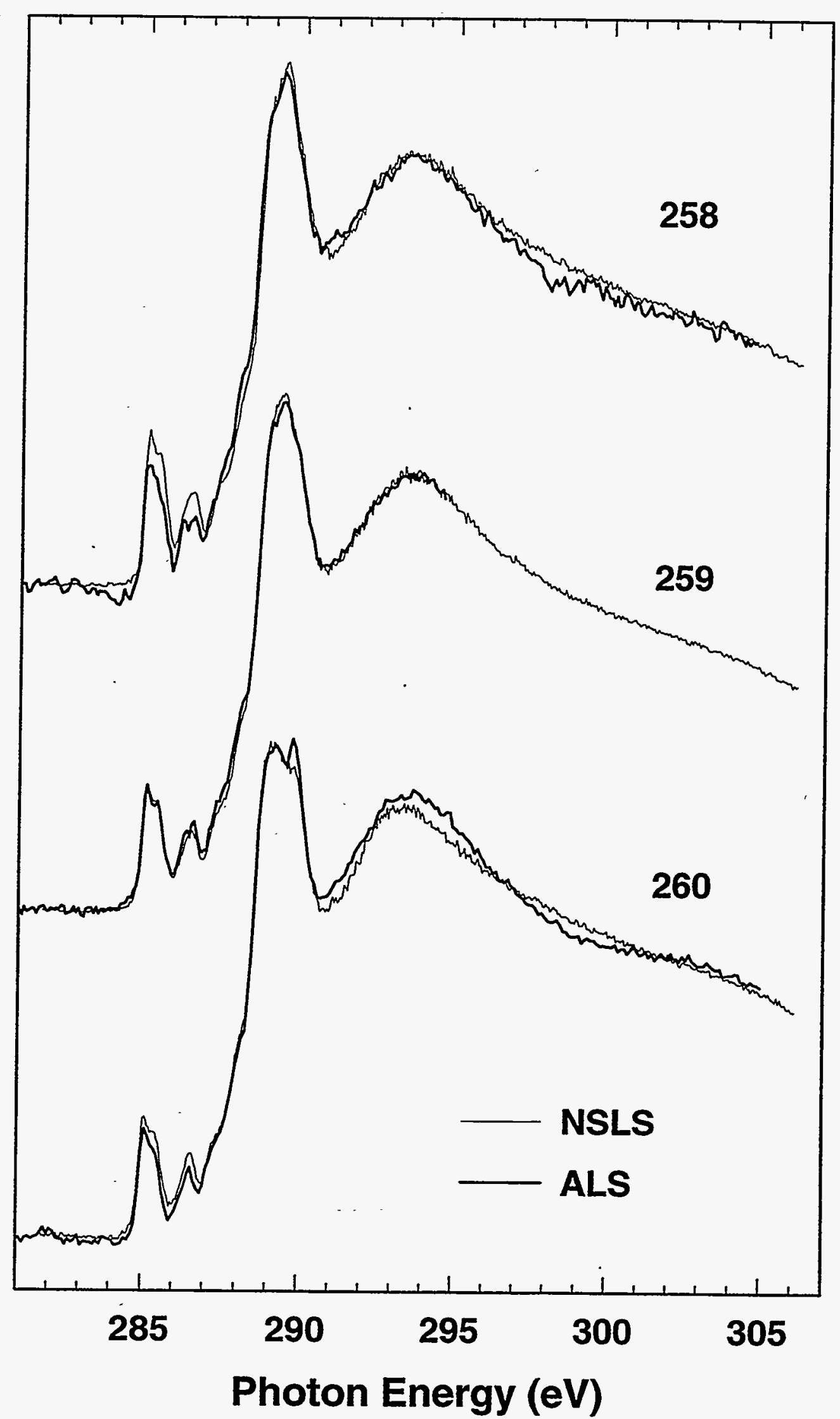

Figure 6 


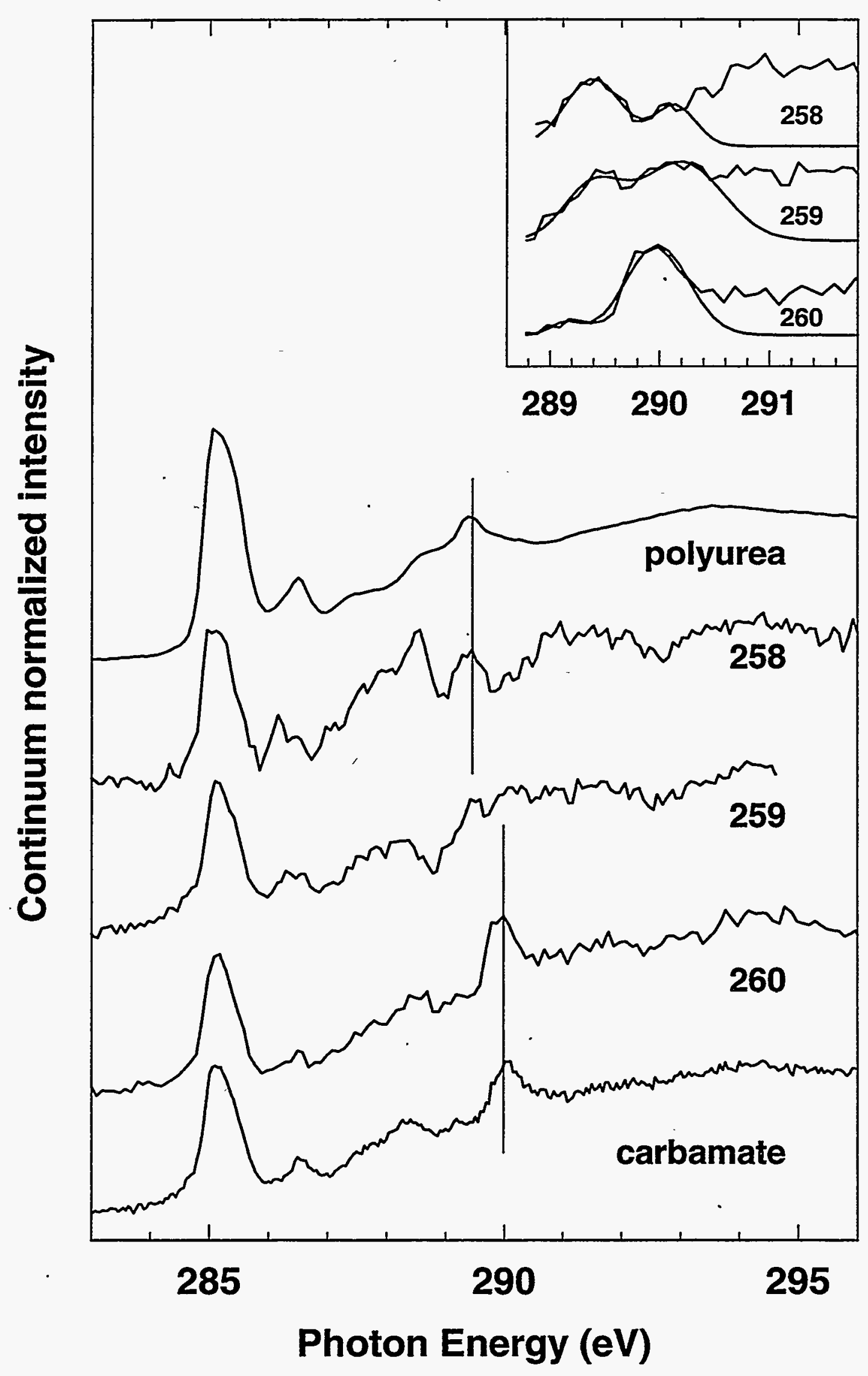

Figure 7 


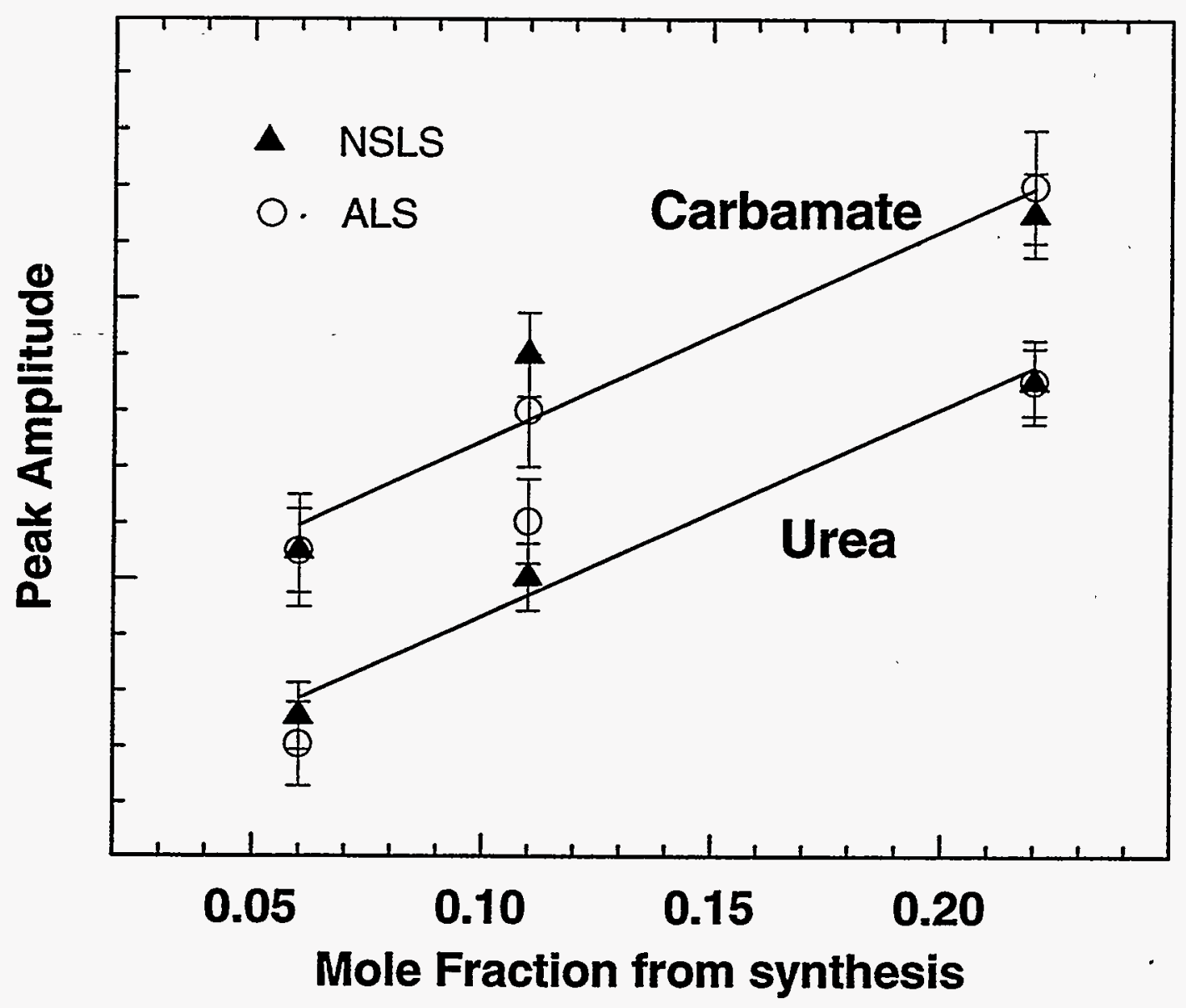

Figure 8

jel-als3.spw 6-Aug-96 


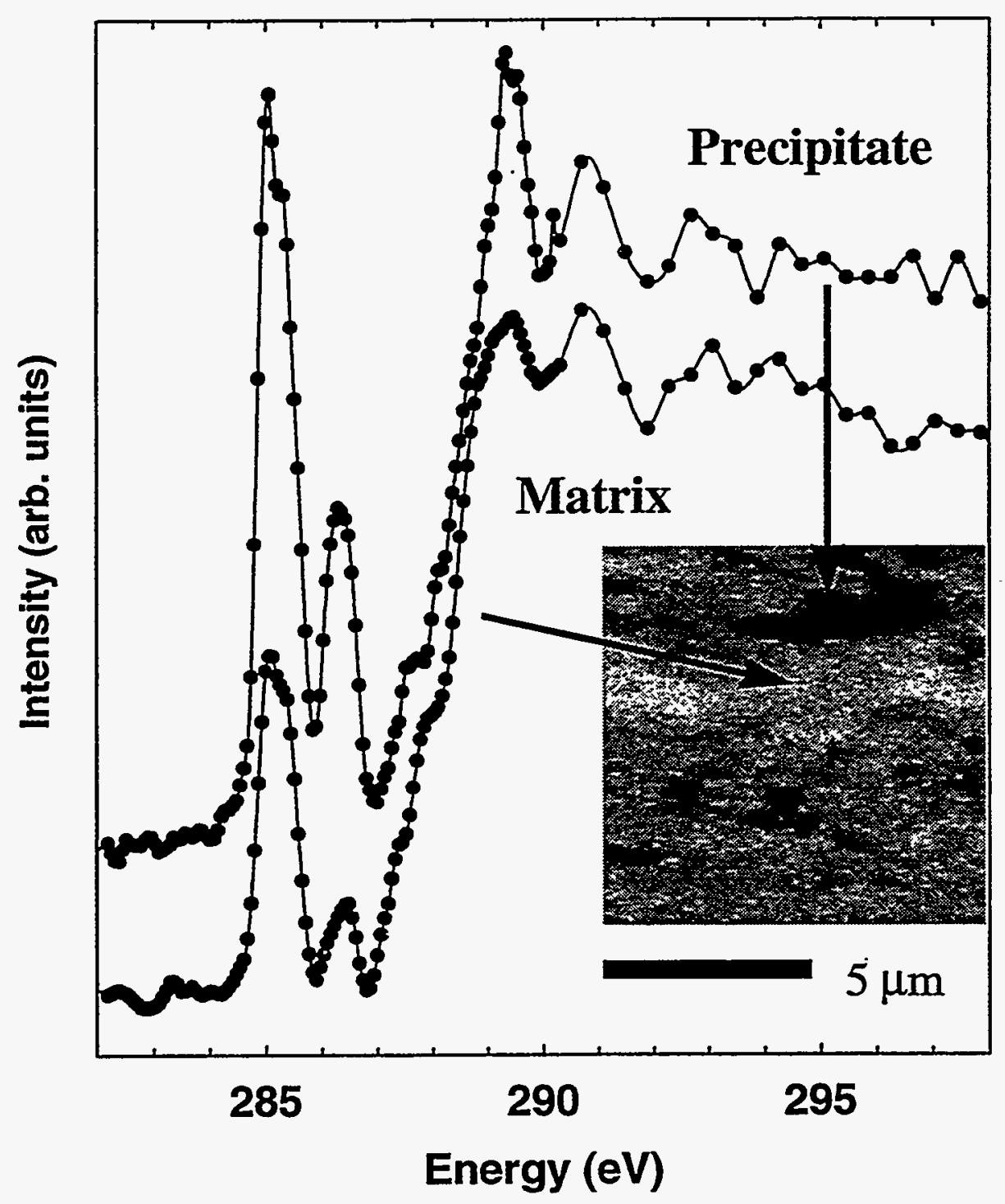

Figure 9 


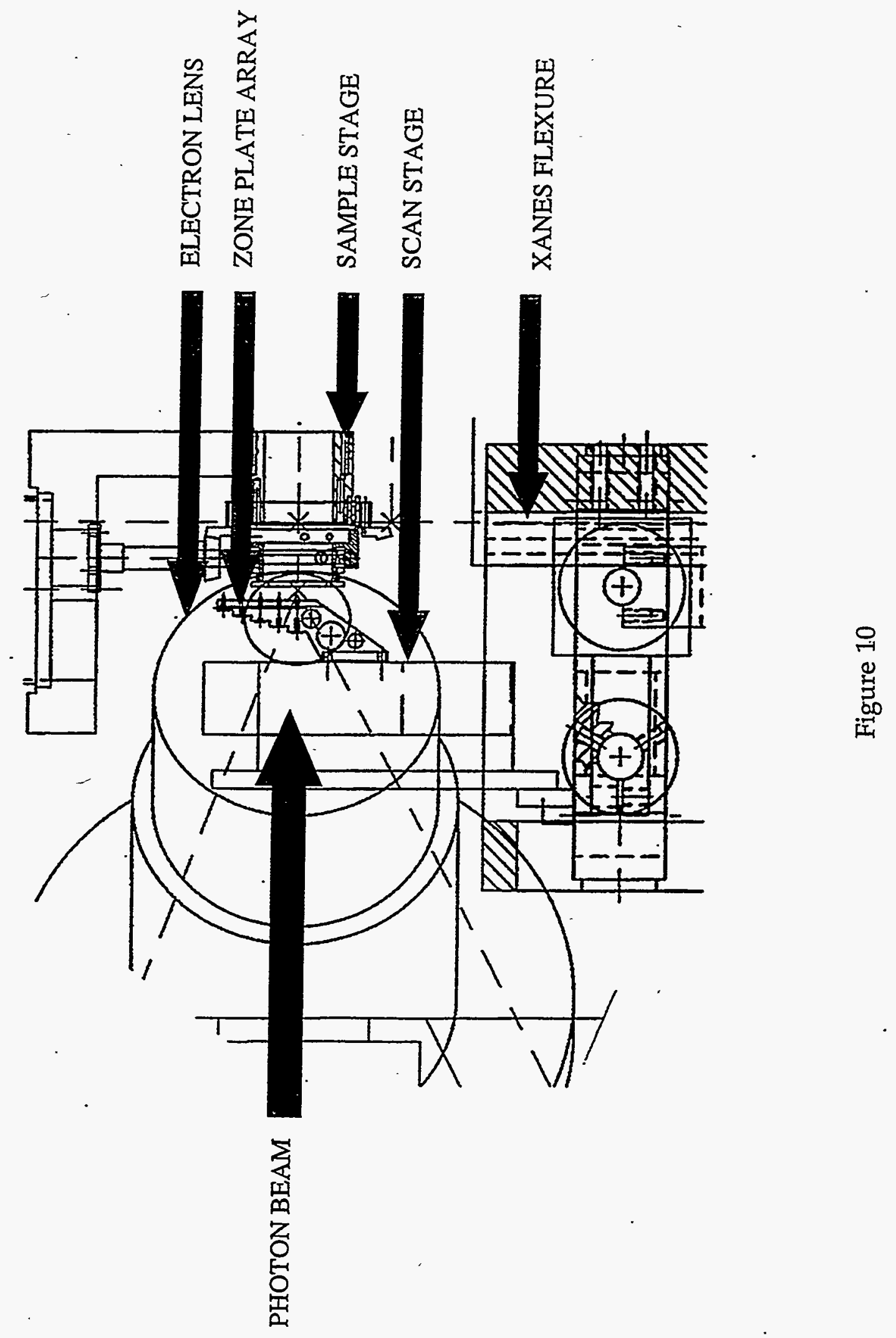




\section{NIST standard}

Courtesy of C.R.Brundle and SEMATECH
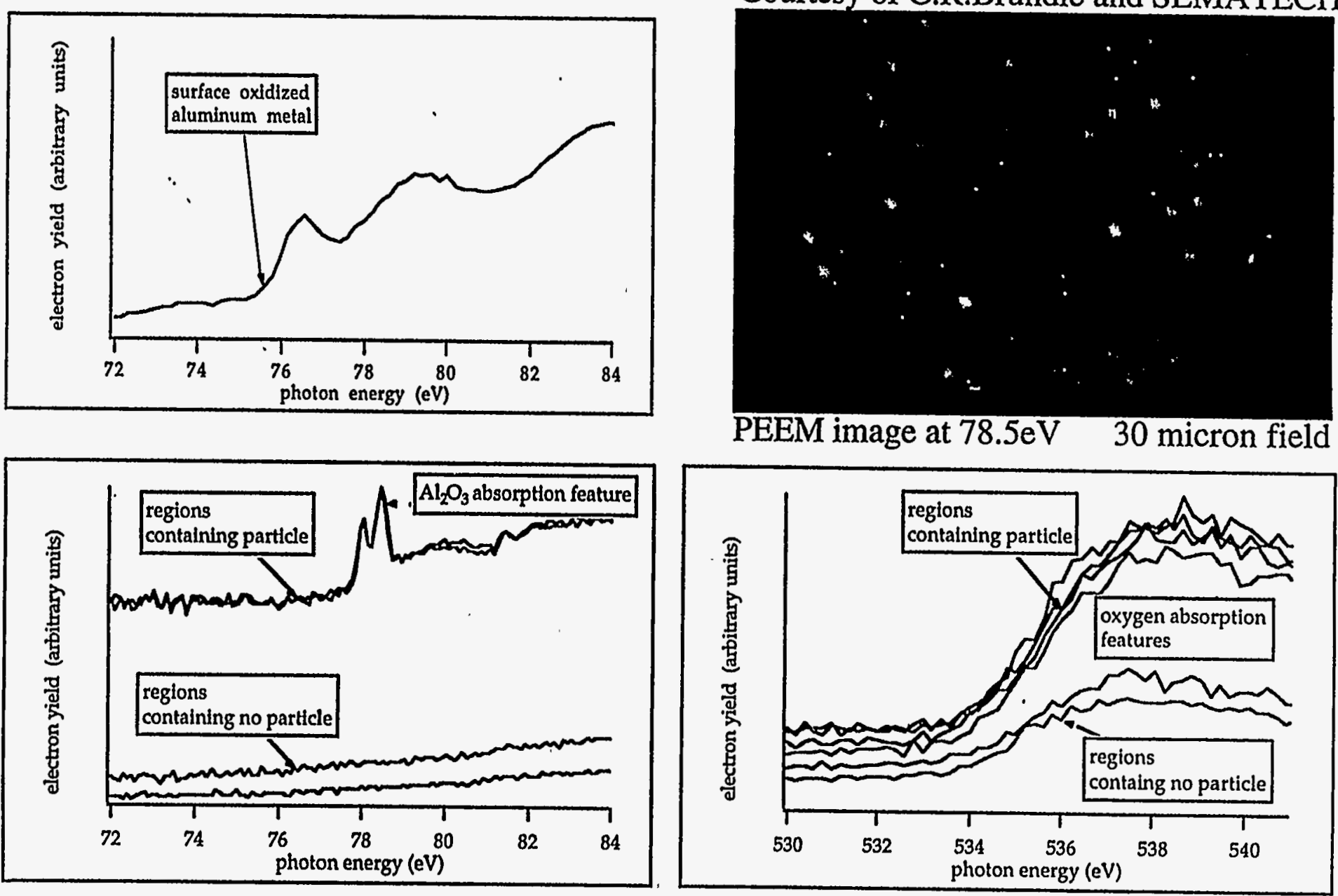

Figure 11 


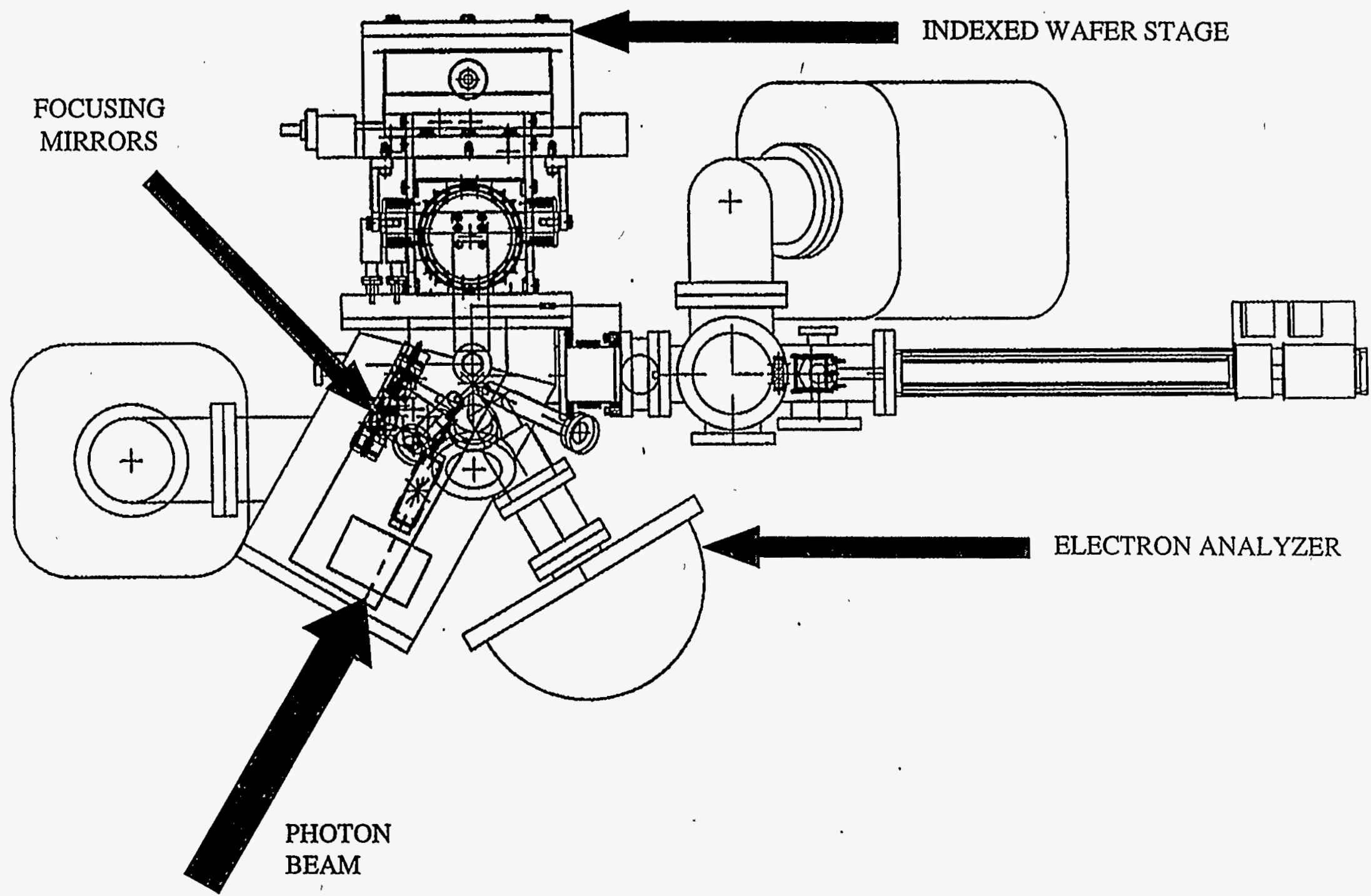

Figure 12 